Revista Calidad en la Educación Superior

Programa de Autoevaluación Académica Universidad Estatal a Distancia, Costa Rica

ISSN 1659-4703

revistacalidad@uned.ac.cr

\title{
LA AUTORREGULACIÓN EN EL PROCESO DE ENSEÑANZA Y APRENDIZAJE: PERCEPCIÓN DE LOS ESTUDIANTES DE LA CÁTEDRA DE TURISMO SOSTENIBLE DE LA UNED
}

\author{
SELF-REGULATION IN THE TEACHING AND LEARNING PROCESS: \\ PERCEPTION OF THE STUDENTS FROM THE CHAIR OF SUSTAINABLE \\ TOURISM AT UNED.
}

\author{
Julia Pérez Chaverri ${ }^{1}$ jperez@uned.ac.cr \\ Susan Solís Rosales ${ }^{2}$ ssolis@uned.ac.cr \\ Universidad Estatal a Distancia, Costa Rica
}

\author{
Volumen 7, Número 1 \\ Mayo 2016 \\ pp. $148-174$
}

Recibido: 30 de agosto, 2015

Aprobado: 13 de marzo, 2016

\footnotetext{
${ }^{1}$ Magister en educación con énfasis en la enseñanza del español S.L. Magister en educación con énfasis en docencia universitaria, profesora de educación especial, profesora capacitadora en la UNED, diseñadora y facilitadora de cursos y talleres virtuales. Correo electrónico jperez@uned.ac.cr

${ }^{2}$ Magister Scientiae en Desarrollo Económico Local con énfasis en Turismo Comunitario de la Facultad Latinoamericana de Ciencias Sociales (FLACSO-CR) y el Instituto Tecnológico de Costa Rica (TEC). Actualmente, directora de la Cátedra Turismo Sostenible de la Escuela de Ciencias Sociales y Humanidades de la UNED-CR. Correo electrónico: ssolis@uned.ac.cr.
} 
La autorregulación en el proceso de enseñanza y aprendizaje: percepción de los estudiantes de la Cátedra de Turismo Sostenible de la UNED

Julia Pérez Chaverri, Susan Solís Rosales

\section{Resumen}

Este avance de investigación expone algunos de los resultados que evidencian cómo un grupo de estudiantes de la Universidad Estatal a Distancia (UNED) perciben sus propios procesos de autorregulación como parte de la estrategia de enseñanza-aprendizaje en las asignaturas: Técnicas de Animación Turística (5166) y Técnicas de Servicio de Alimentos y Bebidas (5163) de los grados de Diplomado y Bachillerato que conforman la cátedra de Turismo Sostenible, perteneciente a la carrera Gestión Turística Sostenible de la Escuela de Ciencias Sociales y Humanidades (ESCH). La presente investigación inició en el I cuatrimestre del 2015 y actualmente está en proceso. Se realiza con una metodología mixta; de esa manera, se puede cuantificar y describir algunas acciones que asume el estudiante para lograr el éxito de las demandas de cada asignatura. Esto le permite a la cátedra tomar decisiones para la mejora continua de los procesos didácticos, pedagógicos y evaluación de los aprendizajes de acuerdo a lo estipulado en el Modelo Pedagógico de la UNED, y sobre todo, favorecer la autorregulación como parte fundamental del proceso de aprendizaje individual que realiza cada persona y que exalta el actual Plan de Desarrollo Académico (2012-2017).

Palabras clave: Autorregulación, metacognición, modelo pedagógico de la UNED, centralidad de la persona estudiante, proceso de enseñanza y aprendizaje.

\section{Summary}

This article is an improvement of results that show how a group of students from the Universidad Estatal a Distancia (UNED) perceive their own processes of selfregulation as part of the strategy of teaching and learning in the subjects: Tourist Animation Techniques (5166) and Food and Beverage Service Techniques (5163) from the Diploma and Bachelor degrees that make up the chair of Sustainable Tourism, part of the Sustainable Tourism Management career at the School of Social Sciences and Humanities (ESCH). This investigation began in the first quarter of 2015 and is currently in process. It is done with a mixed methodology; that way, you can quantify and describe some actions that the student takes to succeed on the demands of each subject. This allows the chair to make decisions for continuous improvement of teaching processes and learning assessments in accordance with the stipulations of the pedagogical model of the UNED, and especially promote self-regulation as a fundamental part of the individual learning process that each person makes, exalting the current Academic Development Plan (2012-2017).

Keywords: Self-regulation, metacognition, pedagogical model of the UNED, centrality of the individual student, teaching and learning process. 


\section{Introducción}

\subsection{El problema y su justificación}

La Universidad Estatal a Distancia (UNED) desde sus inicios, en 1977, ha procurado democratizar la educación (UNED, 2005). La modalidad a distancia se concibió como respuesta a las necesidades educativas, sociales y culturales que presentaba el contexto nacional en aquel momento. Sin embargo, hoy no se puede decir que la universidad realiza su labor académica con los mismos parámetros con los que inició hace más de 35 años.

Dos hechos importantes han marcado la manera de hacer "distancia". Uno es la incorporación de diversos recursos tecnológicos en las acciones docentes (como por ejemplo, el uso de plataformas virtuales de aprendizaje, la dinámica de las asignaturas y la producción de contenidos en formatos que van más allá del texto escrito). Y otro, la creación del Modelo Pedagógico de la UNED en el 2004, el cual permite perfilar qué se espera de un profesional docente o académico y qué se espera del estudiantado.

Por consiguiente, dicha modalidad a distancia no ha sido estática (García, s.f), sino que ha ido evolucionando de acuerdo a las demandas sociales, académicas, tecnológicas y estudiantiles, entre otros factores. Tal complejidad se puede ver, por ejemplo, cuando:

- Existen asignaturas que, por su naturaleza, presentan algún componente presencial obligatorio (CECED, 2015).

- La persona estudiante recibe la información y los contenidos de la asignatura en diferentes formatos y ya no solo un "paquete de textos impresos" (Contreras, s.f)

- Se espera que la persona estudiante, además de estudiar para un examen, deba 
La autorregulación en el proceso de enseñanza y aprendizaje: percepción de los estudiantes de la Cátedra de Turismo Sostenible de la UNED

Julia Pérez Chaverri, Susan Solís Rosales

presentar tareas y realizar una gran variedad de actividades, sincrónicas y asincrónicas, individuales y colaborativas.

- Se espera que la persona estudiante participe activamente no solo en su propio proceso de aprendizaje, sino que además sea co-responsable de la evaluación de esos aprendizajes. Es decir, que sea consciente de cómo aprende y lo que necesita para aprender (CECED, 2014).

Por lo tanto, si desde el 2004 se resalta la centralidad del estudiantado en el Modelo Pedagógico, no basta con pensar y reflexionar sobre cómo se lleva a cabo la estrategia de enseñanza: también es relevante, pertinente y oportuno conocer el modo en que la persona estudiante, desde su punto de vista, asume las demandas de cada asignatura. Así pues, para el I cuatrimestre del 2015, la cátedra de Turismo Sostenible de la Escuela de Ciencias Sociales y Humanidades $(\mathrm{ECSH})$ inició con un proceso de investigación exploratoria con la cual se espera conocer cómo, actualmente, los estudiantes perciben y realizan procesos de autorregulación para cumplir con éxito las demandas que se disponen en un proceso educativo a distancia tan complejo como el que se realiza en la UNED de Costa Rica.

Para llevar a cabo este avance de investigación, se tomó como punto de inicio las respuestas ofrecidas por un grupo de 20 estudiantes que se encuentran avanzados en la carrera (tienen experiencia en el sistema educativo la UNED) y que para el II cuatrimestre del 2015 matricularon una de las asignaturas que ofertó la cátedra:

-Técnicas de Animación Turística (uso de plataforma virtual como complemento) -Técnicas de Servicio de Alimentos y Bebidas (se oferta en modalidad 100\% virtual) 
Debido a que esta investigación está en proceso, solo se presentarán algunos de los resultados alcanzados con la muestra de estudiantes antes mencionada. Estos hacen referencia a:

I Parte: datos generales (género, edad, años de estudio en la UNED, cantidad de asignaturas aprobadas, cantidad de asignaturas reprobadas y abandonadas)

II Parte: datos sobre su proceso de autorregulación (grado de autorregulación pasado y actual, acciones para autorregularse en función de los objetivos, contenidos y actividades de las asignaturas, y evidencias del aprendizaje)

Así que esta investigación es de vital importancia por las siguientes razones:

1. Para el estudiantado: porque se podrá determinar cómo ellos perciben su propio proceso de autorregulación y cómo perciben las asignaturas, de forma tal que estas les estén facilitando (o no) ese quehacer estudiantil. No se debe dejar de lado que cuando los estudiantes se gradúan, una característica que los identifica frente a todos los demás estudiantes de otras universidades, es su capacidad de gestionar su propio aprendizaje, su capacidad de tomar decisiones de manera autónoma su capacidad de evaluar sus aportes, virtudes, limitaciones, sin la necesidad de depender de los demás (o de sus superiores).

2- Para esta disciplina: porque los contenidos y las actividades deben promover personal capacitado para atender las necesidades del sector, además de que la carrera se encuentra inserta en la $\mathrm{ESCH}$; entonces, se pretende que la persona estudiante sea un agente de cambio en su contexto según posibilidades y ámbitos culturales

3. Para la cátedra: porque desarrolla una política de mejora continua, en la cual se aplican instrumentos de observación durante las diversas actividades dentro del aula virtual $u$ otros espacios que se generen. Es necesario pues el estudiante es el centro del modelo pedagógico, y su 
percepción permite hacer ajustes pertinentes y oportunos en las estrategias didácticas de la asignatura.

4. Para la UNED: porque se requieren más estudios que profundicen sobre lo que es autorregulación en un modelo a distancia que indica estar centrado en el estudiante. Dichos fundamentos teóricos, aunque son muy valiosos, no necesariamente se llevan a la práctica. Por ende, es fundamental reflexionar si la universidad organiza actividades desde un punto de vista conductual o tradicional. Se debe analizar si realmente podemos asegurar que desde las cátedras se están gestionando procesos de enseñanza-aprendizaje que les permitan a los estudiantes desarrollar procesos de autorregulación como ejes fundamentales para el aprendizaje y la evaluación.

\section{Marco teórico}

\subsection{Del modelo pedagógico al Plan de Desarrollo Académico}

Desde la creación del Modelo Pedagógico de la Uned en el 2004, se abre un panorama educativo que promueve un proceso de enseñanza y aprendizaje centralizado en el estudiante. Implica que todo el quehacer docente debe planificarse, coordinarse e implementarse de tal forma que la persona que aprende tenga la posibilidad de gestionar su propio proceso formación (UNED, 2004).

Cuando un proceso educativo se centra en el estudiante, asume que dicha persona construye sus conocimientos a partir de sus propias experiencias y la reflexión que hace respecto de ellas (UNESCO, 1988). Por lo tanto, sería contradictorio plantear las estrategias de aprendizaje solamente desde el punto de vista del equipo docente, como si estos tuviesen toda la información y experiencia 
necesaria para saber lo que a cada uno de los estudiantes les interesa, realizan y requieren.

En la autorregulación, quien aprende lleva a cabo ese proceso de manera activa, sistemática, consciente y voluntaria, de modo que desarrolle la capacidad y sobre todo el compromiso de planificar, supervisar, controlar y evaluar sus propios aprendizajes. De ahí que establece las metas que quiere seguir y las estrategias que puede usar para alcanzarlas (Hernández y Pérez, 2014).

Desde el modelo pedagógico de la UNED, el discente no se concibe como un sujeto pasivo que se conforma con recibir y reproducir la información (UNED, 2004). Tal como indica Murillo (2013): "En una institución como la UNED, la enseñanza se lleva a cabo a distancia, pero el aprendizaje lo emprende Usted, como estudiante, aquí y ahora: en el momento en que toma sus materiales didácticos y realiza el esfuerzo por comprenderlos y aprehenderlos" (p.29).

Aunado a lo anterior, es fundamental tener presente que en un proceso de enseñanza y aprendizaje, va más allá de la simple adquisición de contenidos en el que la persona, se concentra en repetir o parafrasear la teoría. En nuestro modelo educativo a distancia, el individuo se caracteriza por adquirir:

- "Competencias intelectuales, sociales, personales y profesionales.

- Habilidades para encontrar, seleccionar y transformar información en conocimiento relevante para la formación personal y la transformación social.

- Ética personal y compromiso social" (UNED, 2004, p.12).

La educación es todo un reto y si se desarrolla en un modelo "sin presencialidad" se hace más complejo el proceso. Por lo que antes se ha dicho, se deben establecer estrategias didácticas y diversos lineamientos políticos que permitan 
La autorregulación en el proceso de enseñanza y aprendizaje: percepción de los estudiantes de la Cátedra de Turismo Sostenible de la UNED

Julia Pérez Chaverri, Susan Solís Rosales

dar sustento y orientación al cuerpo docente e institucional para cumplir con el mandato del modelo de educación a distancia.

Recientemente, se elaboró el Plan de Desarrollo Académico UNED 2012-2017, que se enfoca en el desarrollo de la autorregulación, al indicar que:

El desarrollo de las estrategias de autorregulación del aprendizaje ha de ser objeto de especial atención, y no pueden ni suponerse de antemano, ni pretender que surjan espontáneamente, ni tratarse como contenidos de un curso de metodología o hábitos de estudio a distancia. Han de ser una preocupación transversal, incorporadas en el diseño de los materiales y en la acción tutorial, sobre todo en los períodos iniciales de cada programa. (2013, p. 42)

Para lograr lo anterior, la universidad y especialmente la academia deben garantizar que se ofrecerá una orientación de calidad y excelencia a todos los programas y a los estudiantes que son parte de las ofertas académicas. De esa forma, todos los sectores que conforman la universidad diseñarán y ofrecerán, de manera coordinada, procesos formativos que apunten hacia la autodirección, la autonomía y la autoevaluación. (UNED, 2004).

\subsection{Entre la metacognición, la estrategia de aprender y la autorregulación}

La autorregulación es la capacidad de la persona estudiante para determinar que rutas, medidas, estrategias o materiales requiere para aprender. Es una especie de termómetro que le indica cómo desarrollar su curva de aprendizaje y ante que contextos se puede complejizar la misma. Razón por la cual, toma las previsiones del caso para superar y alcanzar la meta de aprendizaje requerido. 
Por otra parte, la manera en la que se conciba a la persona estudiante dentro de un proceso educativo, determinará la forma en la que se construye el acto educativo: también repercute en la percepción que la persona estudiante tenga de sí mismo dentro de ese proceso de enseñanza y aprendizaje. Tener esta conciencia sobre su propio proceso de aprendizaje, es fundamental para lograr un modelo pedagógico centrado en el estudiante.

Así que además de la autorregulación, se debe promover la metacognición, que según Bordas y Cabera (2001) es:

Aquella habilidad de la persona que le permite tomar conciencia de su propio proceso de pensamiento, examinarlo y contrastarlo con el de otros, realizar autoevaluaciones y autorregulaciones. Es un "diálogo interno" que nos induce a reflexionar sobre lo quéhacemos, cómo lo hacemos, y por qué lo hacemos. (p. 8)

Bordas y Cabrera también afirman que la metacognición debe ser parte del proceso de evaluación de los aprendizajes: de esa forma, el estudiante será consciente de sus avances, sus limitaciones, sus aciertos y desaciertos. Por eso, la evaluación es un mecanismo que en las manos del estudiante no solo le permite evidenciar aprendizajes, sino percatarse de la calidad de esos aprendizajes y regular dichos procesos (2001).

No se puede negar que en un proceso de aprendizaje, muchos son los factores que intervienen para que sea exitoso o no. Según Murillo (2013) tanto el proceso de aprendizaje como el proceso de metacognición y autorregulación estarán determinadas por cuatro áreas que debe desarrollar (autodiagnóstico) la persona estudiante:

1. Cognitiva: procesos de construcción, organización y recuperación del conocimiento. Incluye comprensión, análisis, observación, interpretación, asociación, clasificación, memorización, comprensión de lectura. 
2. Motivacional y afectiva: emociones, estados de ánimo, sentimientos, sensaciones, motivos para estudiar. Incluye el interés, saber por qué se estudia, sensación de éxito y logro, actitud positiva.

3. Compartamental: actos, hábitos, gestos, acciones, respuestas y reacciones. Incluye el adecuado uso del tiempo, la capacidad de organización.

4. Contextual: entorno físico, relaciones interpersonales, contexto cultural, educación previa. Incluye la organización del espacio físico, el entorno de estudio y la creación de relaciones favorables (amigos y grupos de estudio. (pp. 29-30)

Así las cosas, para que la persona estudiante asuma las demandas de una asignatura, debe ser consciente de cuáles son sus características o condiciones personales y, además, de lo que esa demanda le hace sentir y pensar. De forma tal que en tanto una actividad puede estimular o favorecer, otras pueden inhibir o desmotivar al discente.

En consecuencia, aunque la persona estudiante tenga un enfoque de aprendizaje identificado con antelación, puede ser que al desarrollar alguna tarea particular y dependiendo del contexto, deba replantear ese enfoque por uno que le permita enfrentar la tarea.

Los autores De la Fuente, Pichardo, Justicia y Berbén (2008) mencionando a Biggs (1998) indican que existen tres tipos de enfoques que le permiten a la persona estudiante, alcanzar en mayor o menor medida, el éxito académico. En ese sentido, el éxito de un estudiante a propósito de la asignatura puede ser determinado por los siguientes enfoques: 
La autorregulación en el proceso de enseñanza y aprendizaje: percepción de los estudiantes de la Cátedra de Turismo Sostenible de la UNED

Julia Pérez Chaverri, Susan Solís Rosales

Cuadro 1. Enfoques que permiten el éxito académico en la persona estudiante

\begin{tabular}{|c|c|c|}
\hline Enfoque superficial & Enfoque estratégico & Enfoque profundo \\
\hline $\begin{array}{l}\text { Lo que mueve el } \\
\text { aprendizaje es el miedo a } \\
\text { fracasar. La persona } \\
\text { realiza el mínimo esfuerzo } \\
\text { requerido para lograr } \\
\text { resultados mínimos. }\end{array}$ & $\begin{array}{l}\text { Lo que le interesa es el } \\
\text { éxito. La persona realiza su } \\
\text { máximo esfuerzo para } \\
\text { obtener los mejores } \\
\text { resultados posibles. } \\
\text { También se llama enfoque } \\
\text { de logro y realza el “yo” y la } \\
\text { autoestima gracias a la } \\
\text { altas calificaciones. }\end{array}$ & $\begin{array}{l}\text { La motivación intrínseca es } \\
\text { determinante. La persona } \\
\text { se interesa por el contenido } \\
\text { y su importancia. Le motiva } \\
\text { comprender lo que aprende } \\
\text { y lo que esto significa. }\end{array}$ \\
\hline $\begin{array}{l}\text { Las estrategias para } \\
\text { aprender se basan en } \\
\text { rutinas, el aprendizaje es } \\
\text { mecánico y repetitivo. } \\
\text { Se reproduce la } \\
\text { información en el momento } \\
\text { requerido. }\end{array}$ & $\begin{array}{l}\text { Las estrategias se orientan } \\
\text { más que todo a planificar } \\
\text { adecuadamente } \\
\text { actividades, esfuerzo y del } \\
\text { tiempo disponible } \\
\text { para conseguir altas } \\
\text { calificaciones }\end{array}$ & $\begin{array}{l}\text { Las estrategias para } \\
\text { aprender se basan en } \\
\text { descubrir el significado de } \\
\text { lo que se aprende y cómo } \\
\text { esto se relaciona con sus } \\
\text { conocimientos previos. }\end{array}$ \\
\hline $\begin{array}{l}\text { Probabilidad para lograr } \\
\text { aprendizajes de calidad: } \\
\text { muy baja o nula. }\end{array}$ & $\begin{array}{l}\text { Probabilidad para lograr } \\
\text { aprendizajes de calidad: } \\
\text { depende de con cual otro } \\
\text { enfoque se complemente } \\
\text { (con el superficial o con el } \\
\text { profundo), esto depende } \\
\text { del contexto particular de } \\
\text { aprendizaje. } \\
\text { Uno de los elementos que } \\
\text { determina ese contexto son } \\
\text { los criterios de evaluación. }\end{array}$ & $\begin{array}{l}\text { Probabilidad para lograr } \\
\text { aprendizajes de calidad: } \\
\text { muy alta }\end{array}$ \\
\hline
\end{tabular}

Fuente: Julia Pérez Chaverri con base en de la Fuente, et al (2008) 
Como se puede notar en el cuadro 1, el enfoque superficial y el enfoque profundo son excluyentes, es decir, no pueden coexistir al mismo tiempo. Ambos se relacionan con la cantidad y la calidad de los aprendizajes; sin embargo, en el superficial, la persona estudiante no está interesada en alcanzar la mayor cantidad de aprendizajes o bien, que estos sean complejos o profundos. En el enfoque profundo, por un lado, la persona estudiante más bien eso es lo que le motiva. Por otro lado, el enfoque estratégico sí está orientado en el logro del mejor rendimiento académico: es decir, en lograr las mejores calificaciones posibles. De esa forma, podría ser que este enfoque estratégico se combine con alguno de los otros dos. Los autores indican que estos enfoques de aprendizaje se caracterizan por la influencia del proceso metacognitivo como elemento mediador entre la intención o motivo del estudiante y la estrategia de aprendizaje utilizada para estudiar.

Aunque existen investigaciones que evidencian la relación entre estrategias metacognitivas y los enfoques de aprendizaje, no existen tantos estudios para determinar la relación entre el enfoque y los procesos de autorregulación. Sin embargo De la Fuente, J., Pichardo, M., Justicia, F. y Berbén, A. (2008) encontraron la siguiente relación:

La persona presentó un enfoque profundo asociado a estrategias de autorregulación, como resultado mejoró el rendimiento académico.

La persona presentó un enfoque superficial pero con regulación externa, como resultado se mejoró el rendimiento académico.

Esto parece evidenciar que, en términos de rendimiento académico, se pueden obtener buenos resultados aunque la persona no tenga un adecuado proceso de autorregulación. En ese caso, tal proceso podría ser sustituido por una proceso adecuado de regulación; es decir, por la acción docente.

Lo anterior podría evidenciar que si se pretende promover en los estudiantes la capacidad de autorregularse, puede afectarse si las demandas de la oferta 
La autorregulación en el proceso de enseñanza y aprendizaje: percepción de los estudiantes de la Cátedra de Turismo Sostenible de la UNED

Julia Pérez Chaverri, Susan Solís Rosales

académica están orientadas hacia un proceso de aprendizaje memorístico, conductista, descontextualizado y desintegrado. Es decir, un estudiante podría ser "exitoso" con su rendimiento académico, aunque no necesariamente tenga la capacidad de autorregular su propio proceso de aprendizaje, aunque no haga procesos metacognitivos, ni sea un participante activo de los procesos de evaluación. O sea, podría ser "exitoso" a pesar de que el acto educativo no esté centralizado en el estudiante.

\section{Marco metodológico}

La investigación se está llevando a cabo con un enfoque mixto y el alcance será exploratorio pues estudiará la percepción del estudiantado sobre los procesos de autorregulación que llevaron a cabo durante el II cuatrimestre del 2015. Esta sistematización le permitirá a la cátedra tomar decisiones y acciones que mejoren los procesos de enseñanza y aprendizaje de las asignaturas.

\subsection{Sobre las personas participantes}

El proceso de investigación contará con tres grupos de participantes, que están organizados de la siguiente manera:

a. Dos tutoras, que trabajaron con la validación de la encuesta.

b. Un grupo de estudiantes a quienes se les aplicó un instrumento (encuesta) para recolectar los datos identificados.

c. Se espera contar con más de la mitad de los estudiantes que matricularon las asignaturas Técnicas de Animación Turística y Técnicas de Servicio de Alimentos y Bebidas, en el II cuatrimestre del 2015.

d. Una muestra de estudiantes, que se seleccionarán para profundizar en algunos detalles que parecen muy relevantes sobre cómo, por qué y para qué realizan procesos de autorregulación. 
La autorregulación en el proceso de enseñanza y aprendizaje: percepción de los estudiantes de la Cátedra de Turismo Sostenible de la UNED

Julia Pérez Chaverri, Susan Solís Rosales

\subsection{Técnicas e instrumentos}

Se elaboró una encuesta para aplicarla en línea y de manera anónima a los estudiantes. Antes de esta parte, primero se realizó la validación con dos tutoras de la cátedra. A dichas personas se les envió el instrumento y ellas lo completaron e hicieron observaciones para mejorar el cuestionario.

Posteriormente, se envió por correo electrónico a todos los estudiantes matriculados en las dos asignaturas que se ofertaron en este II cuatrimestre, así como también se incluyo el instrumento en la plataforma MOODLE que es otro punto de encuentro con la persona estudiante; de este proceso ya se obtuvieron 20 respuestas. Dicho instrumento consta de tres partes: datos personales, datos sobre su proceso de autorregulación y datos sobre la propuesta didáctica de la asignatura.

Eventualmente en el proceso de investigación, se seleccionará una muestra de estos participantes con quienes se llevará a cabo una entrevista semiestructurada, la intensión de esta etapa es clarificar y profundizar en algunos detalles de las respuestas otorgadas en la encuesta y preguntar sobre nuevas inquietudes que surgirán como parte del análisis de la información preliminar. De esta forma, se realizará una triangulación de los datos recopilados a partir de informantes clave, encuesta y entrevista.

Sin embargo, para efectos de esta ponencia, únicamente se tomarán en cuenta los datos de esta primera encuesta con el primer grupo de participantes.

\section{Resultados preliminares}

A continuación, se presentan algunos resultados preliminares tomando como punto de referencia las primeras 20 encuestas recibidas. Aunque el instrumento posee preguntas de diferentes tipos, en este avance solo se presentarán las respuestas relacionadas con: 
La autorregulación en el proceso de enseñanza y aprendizaje: percepción de los estudiantes de la Cátedra de Turismo Sostenible de la UNED

Julia Pérez Chaverri, Susan Solís Rosales

I Parte: datos generales (género, edad, años de estudio en la Uned, cantidad de asignaturas aprobadas, cantidad de asignaturas reprobadas y abandonadas

II Parte: datos sobre su proceso de autorregulación (nivel de autorregulación pasado y actual, acciones para autorregularse en función de los objetivos, contenidos y las actividades de las asignaturas, evidencias del aprendizaje)

En cuanto a los 20 participantes, es posible indicar que 15\% fueron hombres y $85 \%$ fueron mujeres. De ellos, 8 (40\%) personas matricularon la asignatura Técnicas de Servicio en Alimentos y Bebidas, 9 (45\%) Técnicas de Animación Turística y $3(15 \%)$ participantes eran estudiantes de ambas asignaturas. Además, $20 \%$ tiene entre 1 o 2 años de ser estudiante en la UNED, $40 \%$ de ellos tiene 3 o 4 años y otro $40 \%$ tiene 5 años o más. Estos datos representan que $80 \%$ de los participantes de esta encuesta ya tienen un largo recorrido en la UNED, y que de alguna manera, han logrado ir desarrollando una metodología de estudio lo suficientemente congruente con esta modalidad a distancia.

Figura 1.

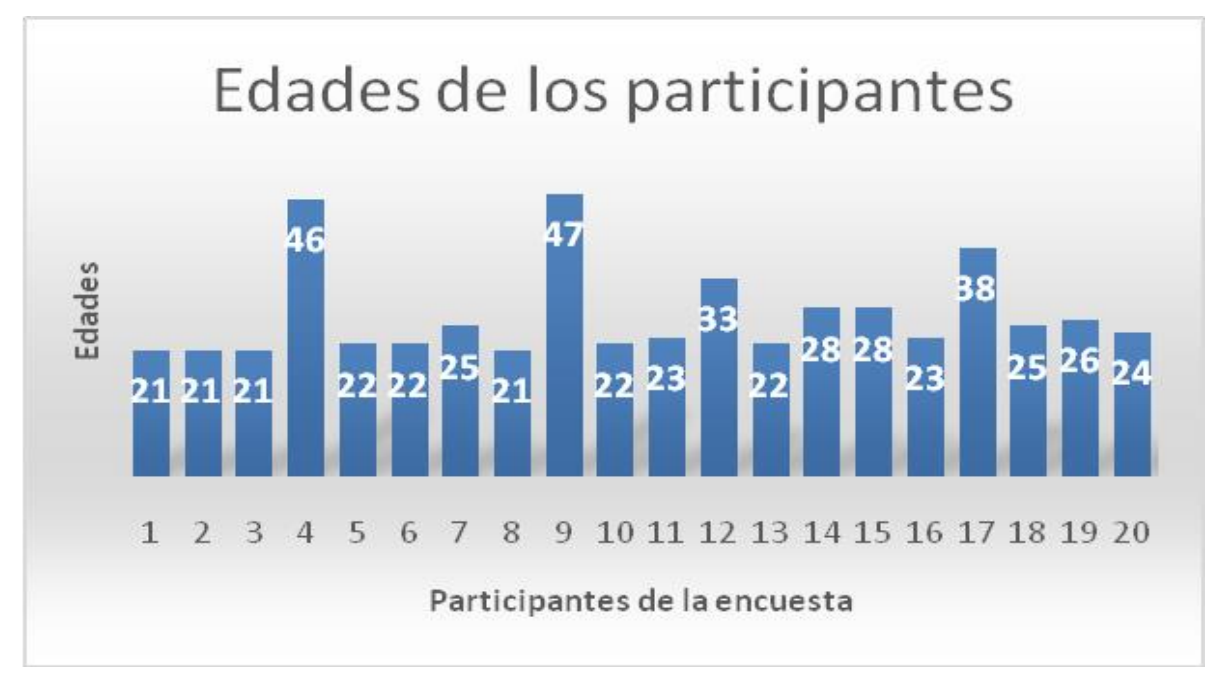

Fuente: Julia Pérez Chaverri y Susan Solís Rosales (2015) 
La autorregulación en el proceso de enseñanza y aprendizaje: percepción de los estudiantes de la Cátedra de Turismo Sostenible de la UNED

Julia Pérez Chaverri, Susan Solís Rosales

Como se observa en la figura 1, las personas con menos edad son de 21 años, mientras que las dos de más edad superan los 45 . Dentro de este grupo, 16 tienen menos de 30 años, 2 tienen edades entre los 30 y 40 años, y los últimos dos son mayores de 45 años; entonces, en su mayoría son jóvenes y jóvenes adultos. Por lo tanto, si anteriormente se indicó que 8 de cada 10 participantes ya tiene 3 años o más estudiando esta carrera, se puede afirmar que esta población inició a una edad muy temprana sus estudios. Posiblemente, terminaron el colegio y escogieron la UNED para hacer sus estudios universitarios. Los estudiantes cada vez son más jóvenes.

Cuadro 2. Cantidad de asignaturas que matricula la persona por cada cuatrimestre

\begin{tabular}{|c|c|}
\hline $\begin{array}{c}\text { Matricula de asignaturas } \\
\text { por cuatrimestre }\end{array}$ & $\begin{array}{c}\text { Porcentaje de } \\
\text { personas }\end{array}$ \\
\hline 4 & $20 \%$ \\
\hline 3 & $55 \%$ \\
\hline 2 & $20 \%$ \\
\hline 5 & $5 \%$ \\
\hline \multicolumn{2}{|l}{ Fuente: Julia Pérez Chaverri y Susan Solís Rosales (2015) }
\end{tabular}

El cuadro 2 representa el promedio de asignaturas que frecuentemente suelen matricular estos participantes. Este cuadro nos indica que el $75 \%$ de los participantes matriculan entre 3 y 4 asignaturas por cuatrimestre, es decir, asumen una carga significativa de estudio, por lo tanto, reafirma la tesis que ya son estudiantes que, de alguna manera, han interiorizado el modelo a distancia de la UNED. 
Figura 2.

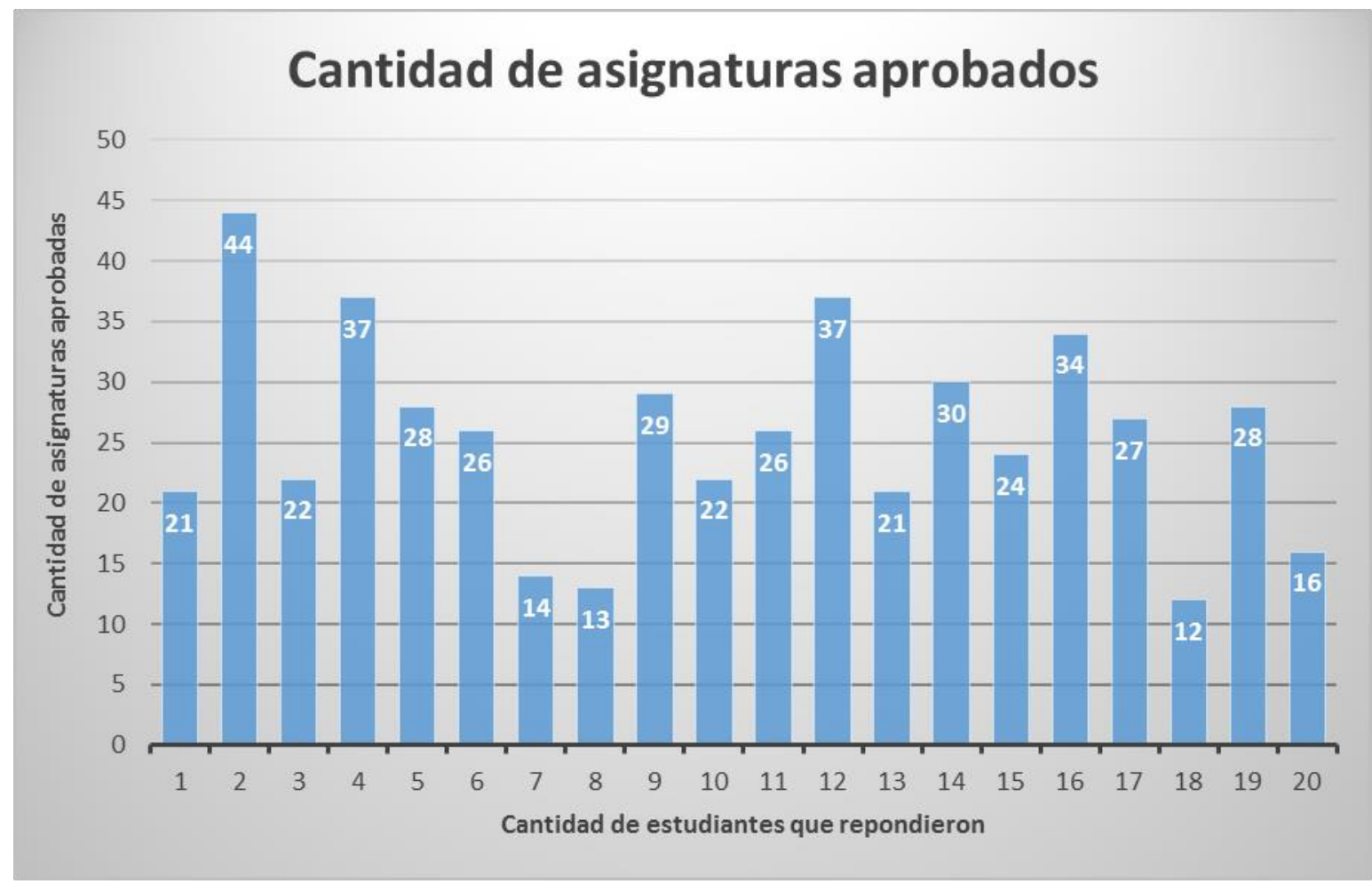

Fuente: Julia Pérez Chaverri y Susan Solís Rosales (2015)

En la figura 2, se evidencian todas las asignaturas que a la fecha tienen aprobadas en la UNED, no necesariamente implica que todas corresponden a la misma carrera, pues existe la posibilidad de que algunos estén haciendo estudios en más de una carrera al mismo tiempo. Sin embargo, ese dato no fue preguntado directamente dentro de esta primera encuesta. En total, estos estudiantes han aprobado una cantidad de 511 asignaturas. 
Figura 3.

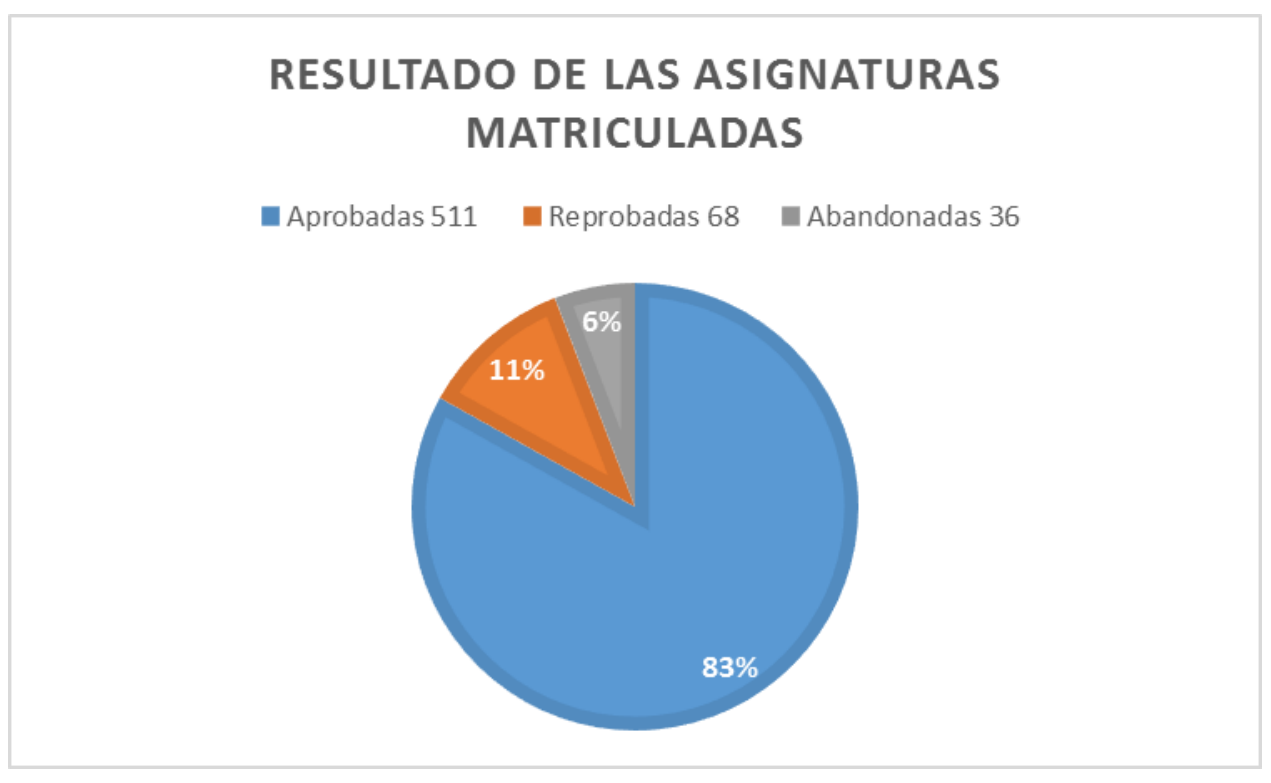

Fuente: Julia Pérez Chaverri y Susan Solís Rosales (2015)

En la figura 3, si comparamos la cantidad de asignaturas aprobadas con el total de asignaturas reprobadas y abandonadas, estos son los resultados. Como se observa, hay una diferencia significativa si comparamos estos datos. Pero no se puede saber si para aprobar una asignatura tuvo que matricularla varias veces. Al revisar los resultados, 19 estudiantes al menos han perdido 1 asignatura, solo 1 estudiante indica que nunca ha perdido ninguna asignatura. Sin embargo, ese mismo estudiante es el que más veces ha abandonado asignaturas: un total de 16. El estudiante que más veces ha reprobado asignaturas es el que indica la cantidad de 11. Un dato que llama la atención en esta encuesta es que estos dos estudiantes con las cantidades superiores en ambos eventos son precisamente los de más edad (46 y 47 años).

II Parte: datos sobre su proceso de autorregulación (grado de autorregulación pasado y actual, acciones para autorregularse en función de los objetivos, contenidos y actividades de las asignaturas, y evidencias del aprendizaje) 
La autorregulación en el proceso de enseñanza y aprendizaje: percepción de los estudiantes de la Cátedra de Turismo Sostenible de la UNED

Figura 4.

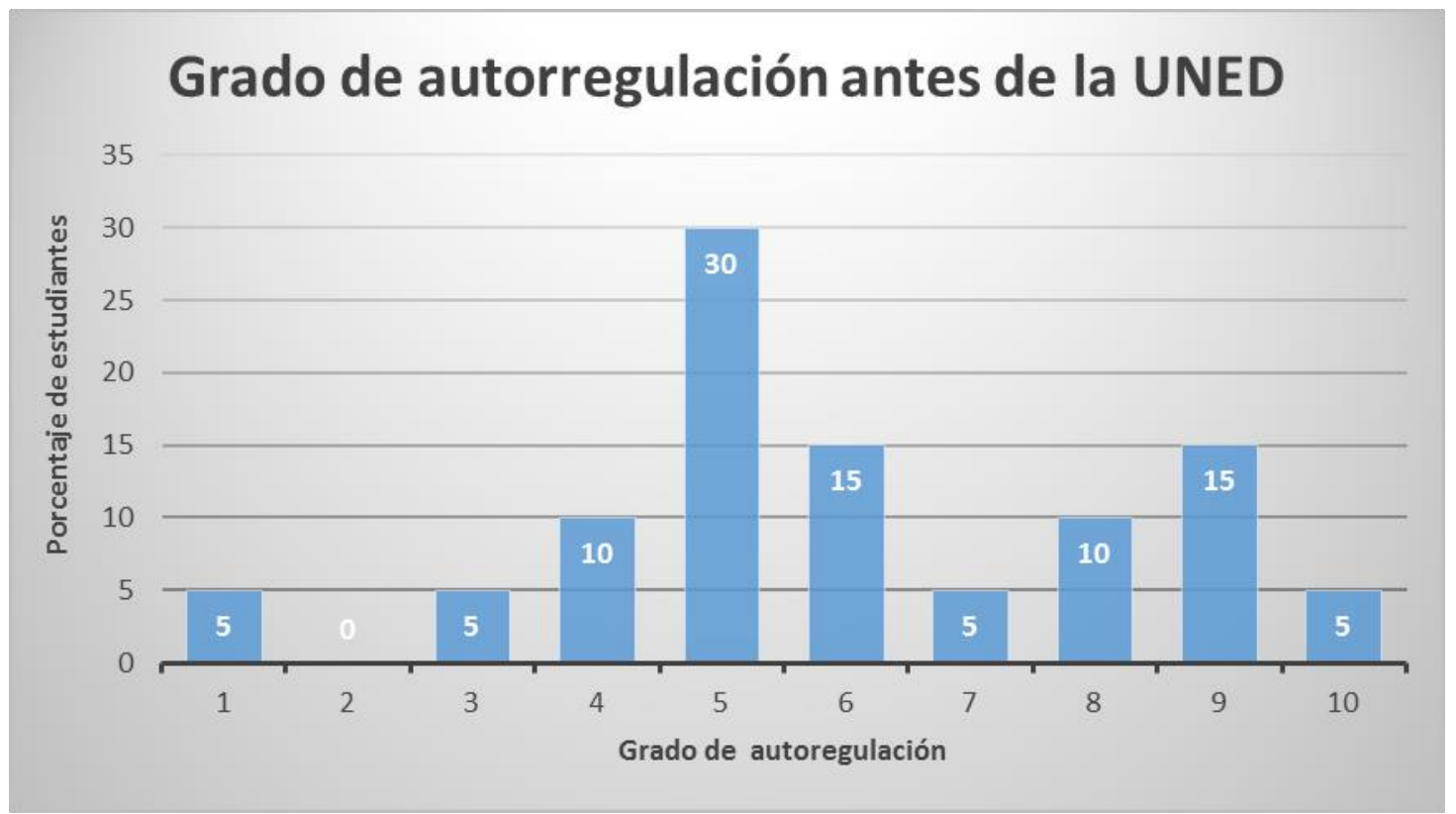

Fuente: Julia Pérez Chaverri y Susan Solís Rosales (2015)

Al preguntarles a los participantes, qué calificación le darían al nivel de autorregulación que poseían antes de ingresar a la UNED, se puede confirmar en la figura 4 que hay puntajes que van desde 1 a 10. La nota que más se repitió fue 5 , seguidamente de 6 y 9.

Figura 5.

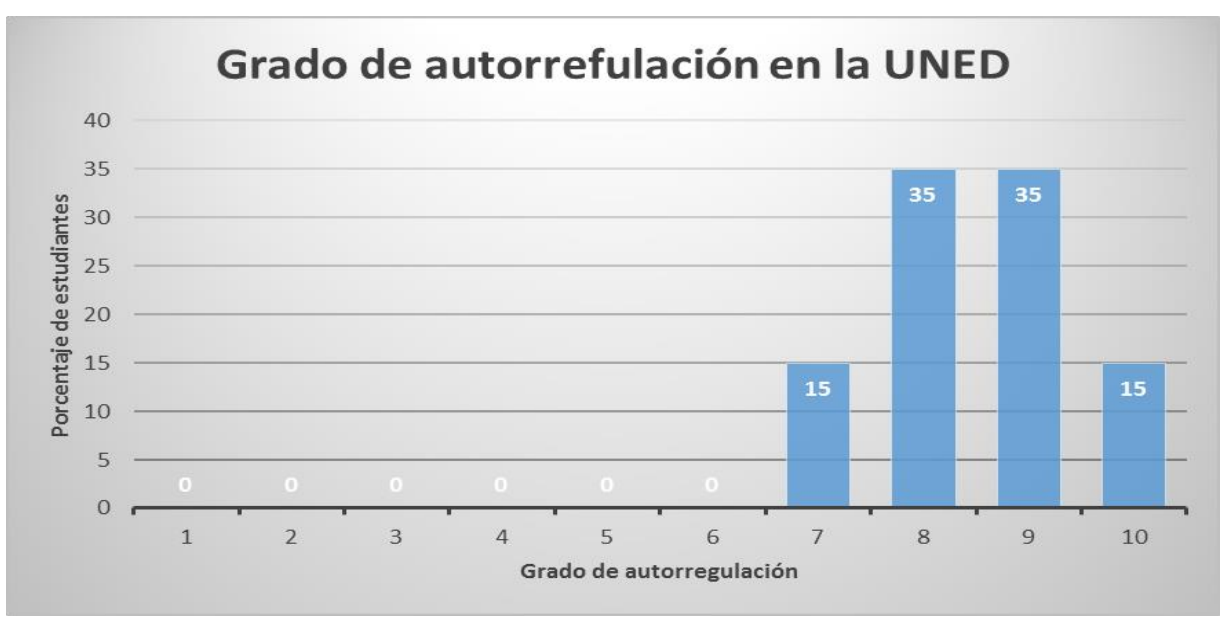

Fuente: Julia Pérez Chaverri y Susan Solís Rosales (2015) 
La autorregulación en el proceso de enseñanza y aprendizaje: percepción de los estudiantes de la Cátedra de Turismo Sostenible de la UNED

Julia Pérez Chaverri, Susan Solís Rosales

Sin embargo, como se puede extraer de la figura 5, la calificación que ahora se otorgan ha aumentado en general. Las dos notas más altas son de 8 y 9 . Sin embargo, al revisar los datos individuales, 4 personas se otorgaron la misma nota, mientras que una persona se otorgó una nota inferior. Queda la duda si antes de entrar a la UNED, la calificación otorgada por ellos mismos refleja que tenían menos capacidad de autorregularse o si más bien el proceso educativo anterior (en el caso de los que recién terminaron el colegio) no les exigía tanto esfuerzo en términos de autorregulación. Dicho de otro modo, no se podría aún determinar que la calificación es buena o mala, sino que es un reflejo del nivel de autonomía o dependencia que promueve el sistema educativo en general.

Otra duda que surge y que se retomará en las etapas siguientes, es si realmente la calificación refleja que han mejorado o mantenido su capacidad de autorregularse o si es que ya después de ingresar en la UNED tienen mayor conciencia de tal proceso. Valdría la pena averiguar si su manera de autorregularse es igual para todas las asignaturas o si con cada una determinan una estrategia de trabajo y organización diferentes; además, hay que indagar si el rendimiento académico de las asignaturas es un reflejo de esa estrategia de autorregulación. 
La autorregulación en el proceso de enseñanza y aprendizaje: percepción de los estudiantes de la Cátedra de Turismo Sostenible de la UNED

Julia Pérez Chaverri, Susan Solís Rosales

Cuadro 3. Acciones relevantes para autorregularse en función de objetivos de aprendizaje, contenidos y actividades propias de la asignatura.

\begin{tabular}{|l|c|c|c|}
\hline Acciones/cantidades & Objetivos & Contenidos & Actividades \\
\hline Elaboración agenda/cronograma & 16 & 1 & 14 \\
\hline Lectura/uso de material & 6 & 13 & 2 \\
\hline Investigación de otras fuentes & 1 & 6 & 5 \\
\hline $\begin{array}{l}\text { Extracción de ideas principales/ } \\
\text { resúmenes }\end{array}$ & 5 & 6 & \\
\hline Estudio & 5 & & 1 \\
\hline Seguimiento de indicaciones & & 3 & 1 \\
\hline Realización prácticas & & 1 & 1 \\
\hline Análisis de los objetivos & & & 7 \\
\hline Realizar los trabajos con tiempo & & & \\
\hline Revisar las fechas constantemente & & 5 & \\
\hline Consulta de dudas con el tutor & 4 & & \\
\hline Motivación & & 3 & \\
\hline Elaboración preguntas y responderlas & & & \\
\hline Seguimiento de instrucciones & & 1 & \\
\hline Revisión de los resultados & & 1 & \\
\hline Uso del método de la tutorías & & & \\
\hline Análisis de información & & & \\
\hline Uso de ejemplos prácticos/ reales & & & \\
\hline Memorización & & & \\
\hline
\end{tabular}

Fuente: Julia Pérez Chaverri y Susan Solís Rosales (2015)

Al preguntarles a las personas participantes que indicaran las dos acciones más importantes para autorregularse en función de los objetivos de aprendizaje, los contenidos y las actividades propias de la asignatura, las respuestas se agruparon en el Cuadro 3: 
Se concluye que varios estudiantes coinciden en que las acciones más importantes son: organizar el tiempo (elaborar el tiempo vrs actividades), leer o revisar los diferentes materiales e investigar en otras fuentes. Se trata de acciones necesarias todas para la autorregulación en función de los objetivos, contenidos y actividades.

En cuanto a los objetivos, solo una persona indica que una de las acciones principales es analizar lo que estos indican. Con estas respuestas no se puede determinar, a ciencia cierta, si los estudiantes conciben al objetivo como un contenido teórico, si lo percibe como una actividad; incluso, no le encuentran ningún tipo de utilidad.

Otro elemento por resaltar es que varios estudiantes mencionan que también buscan información en otros medios, además de los que les ofrece la UNED. No se conforman con los contenidos oficiales, sino que algunos sienten la necesidad de complementarlos con otros puntos de vistas de otros autores o fuentes. Sería interesante preguntarles a qué se debe esa acción: si es porque les interesa profundizar más, porque sienten que los contenidos del curso no son suficientes o porque no les queda completamente claro y requieren de clarificación.

Una de las personas mencionó como acción importante en relación con los contenidos, relacionar la teoría con ejemplos o situaciones reales. Precisamente, esta persona a la fecha no ha perdido ni abandonado ninguna asignatura; de hecho, hasta julio del 2015 cuenta con 26 aprobadas. Por eso, cuando se continúe con la investigación, será necesario profundizar en la relación de las acciones de autorregulación con la calidad de sus aprendizajes, el rendimiento académico y su enfoque de aprendizaje. Asimismo, valdría la pena averiguar si esta acción la solicita la asignatura o si es una estrategia muy personal que le da buenos resultados. 
La autorregulación en el proceso de enseñanza y aprendizaje: percepción de los estudiantes de la Cátedra de Turismo Sostenible de la UNED

Julia Pérez Chaverri, Susan Solís Rosales

Otra respuesta destacable es el estudiante que aduce que una acción importante para autorregulación es la memoria. Hasta julio del 2015 cuenta con 37 asignaturas aprobadas, 6 perdidas y 2 abandonadas. Queda la duda, si efectivamente esa acción siempre la ha dado buenos resultados y qué le hace pensar que es una estrategia relevante para su autorregulación.

Otro elemento a resaltar de estas respuestas es que en términos generales no hay diferencias explícitas entre las acciones que realizan los estudiantes cuya asignatura es completamente virtual y los que realizan el proceso educativo a distancia sin virtualidad obligatoria. Es decir, en algunos casos los estudiantes indicaron "realizar la lectura que está en la plataforma", aunque mencionan la plataforma, la acción en sí misma se refiere a "leer", en ese caso lo que cambió fue el formato del texto, pero la acción es la misma. Así que puede ser que para autorregularse, el medio por sí mismo no es lo que determina la capacidad de la persona. 
La autorregulación en el proceso de enseñanza y aprendizaje: percepción de los estudiantes de la Cátedra de Turismo Sostenible de la UNED

Julia Pérez Chaverri, Susan Solís Rosales

Cuadro 4. Formas como la persona estudiante determina logro alcanzar los objetivos de aprendizaje.

\begin{tabular}{|l|c|}
\hline Evidencias del aprendizaje & Respuestas \\
\hline Revisando notas de los exámenes & 9 \\
\hline Cuando tengo claro o comprendo un concepto o tema & 8 \\
\hline Realizar prácticas & 4 \\
\hline Contestando de memoria cuando estudio & 4 \\
\hline Cuando aplico lo estudiando en situaciones reales & 2 \\
\hline Cuando repaso & 2 \\
\hline Comparo los objetivos vrs mi desempeño profesional & 1 \\
\hline Revisando la Ruta de aprendizaje previamente & 1 \\
\hline Conforme avanza el curso & 1 \\
\hline Planificación mensual & 1 \\
\hline Con la aprobación del curso & 1 \\
\hline Comparo mis respuestas del examen con el libro & 1 \\
\hline Cuando hago las tareas & 1 \\
\hline Revisando la plataforma & 1 \\
\hline Con la retroalimentación recibida & 4 \\
\hline
\end{tabular}

Fuente: Julia Pérez Chaverri y Susan Solís Rosales (2015)

Precisamente, el cuadro 4 muestra las respuestas de los estudiantes cuando indican la manera en la que ellos se dan cuenta que ya lograron alcanzar los aprendizajes de la asignatura. Estas son las respuestas:

Como se observa, la mayoría de las respuestas están más orientadas hacia el rendimiento académico o la realización y presentación de las actividades obligatorias de la asignatura, que con el logro de aprendizajes profundos. Al menos no se logra evidenciar mediante las respuestas (o la manera en la que ellos expresan sus ideas). Igualmente, pareciera que depende del facilitador o docente, 
La autorregulación en el proceso de enseñanza y aprendizaje: percepción de los estudiantes de la Cátedra de Turismo Sostenible de la UNED

Julia Pérez Chaverri, Susan Solís Rosales

la confirmación de esos aprendizajes. Así pues, no hay evidencias explícitas de que el estudiante determine o autoevalúe su proceso de aprendizaje personal.

\section{Conclusiones}

Se debe continuar desarrollando diversos aspectos con respecto al proceso investigativo que se está llevando a cabo dentro de la Cátedra de Turismo Sostenible. No obstante, los elementos analizados en este avance permiten:

1. Evidenciar que la capacidad de autorregularse no es cuestión de edad o experiencia universitaria. Sin duda, no todas las personas han logrado desarrollarla o no todos saben cómo mejorar o ajustarlas a las diferentes situaciones dependiendo del contexto en el que se encuentren (como estudiantes o profesionales).

2. Comprender que la autorregulación puede estar ligada con el rendimiento académico exitoso, pero también estos resultados pueden depender de un proceso de regulación y acompañamiento del equipo docente. Por lo tanto, no obligatoriamente todos los estudiantes que logran notas altas o superiores a las notas mínimas lo hacen por su propia capacidad de autogestionar sus estudios y procesos de aprendizaje. Entonces, no estrictamente son personas autónomas.

3. Determinar cuáles limitaciones enfrentaron con las asignaturas reprobadas o abandonadas; es decir, si el motivo de esta situación fue la manera en la que se autorregularon o dónde estuvo la causa de tales resultados.

4. Afirmar que las personas estudiantes de este grupo se perciben como personas autorreguladas. En su mayoría, han logrado mejorar ese proceso. No obstante, falta profundizar en cuán buenos resultados les habrán dado las acciones empleadas en el II cuatrimestre del 2015, específicamente en las asignaturas de Técnicas de Animación Turística (5166) y Técnicas de Servicio de Alimentos y Bebidas (5163).

5. Reflexionar en torno a qué motiva al estudiante cuando inicia un proceso educativo y, en ese sentido, cómo lleva a cabo su estrategia de aprendizaje en función de los requerimientos de esa asignatura (con virtualidad o sin ella). 
La autorregulación en el proceso de enseñanza y aprendizaje: percepción de los estudiantes de la Cátedra de Turismo Sostenible de la UNED

Julia Pérez Chaverri, Susan Solís Rosales

6. Reflexionar cuándo el estudiante siente mayor motivación, cuándo su autorregulación le permite alcanzar un rendimiento académico alto o cuándo le permite alcanzar aprendizajes de calidad o todas las situaciones mencionadas.

7. Valorar si los diversos aspectos que conforman la asignatura, efectivamente promueven procesos de autorregulación, y si están en función de que el estudiante alcance un rendimiento académico alto, aprendizajes calidad o ambos. También se debe reflexionar si la modalidad influye o no en el proceso de autorregulación y en el proceso de aprendizaje de cada estudiante.

A partir de todo lo mencionado, se recomienda hacer una reflexión de cómo mejorar el diseño y la implementación de las asignaturas. En ese sentido, hay que propiciar una estrategia de aprendizaje que favorezca la metacognición y la autoevaluación como estrategias que fundamentan la autorregulación estudiantil en la UNED.

\section{Referencias}

Bordas, M. y Cabrera, F. (enero - abril, 2001). Estrategias de evaluación de los aprendizajes centrados en el proceso. Revista Española de Pedagogía. Año LIX(218), 25-48. Recuperado de http://cmapspublic3.inmc.us/rid=1GLSW84JS-WYZWX0H40/Evaluaci\%C3\%83\%C6\%92\%C3\%82\%C2\%B3n\%20 del\%20Proceso\%2 0de\%20Aprendizaje.pdf

Centro de Capacitación de Educación a Distancia (CECED). (2014). Informe de resultados de las evaluaciones de cursos del CECED. Sin publicación

Centro de Capacitación de Educación a Distancia (CECED). (2015). Informe de matrícula de cursos de capacitación. Sin publicación

Contreras, O. (s.f). Diplomado Nacional "Implementación de Programas Académicos en Educación a Distancia" Diplomado Nacional "Implementación de Programas Académicos en Educación a Distancia" Módulo 2: Elementos Pedagógicos para Programas Académicos en Educación a Distancia Material didáctico y recursos para el aprendizaje en la educación a distancia. Recuperado de http://cvonline.uaeh.edu.mx/Cursos/TecEduc/DCL/LICEDUC MV/seleccion/l ec contreras material didactico.pdf 
La autorregulación en el proceso de enseñanza y aprendizaje: percepción de los estudiantes de la Cátedra de Turismo

De la Fuente, J., Pichardo, M., Justicia, F. y Berbén, A. (2008). Enfoques de aprendizajes, autorregulación y rendimiento en tres universidades europeas. Psicothema, 20(4), 705-711. Recuperado de http://www.unioviedo.es/reunido/index.php/PST/article/view/8719/8583

García, L. (s.f). Educación a distancia: ayer y hoy. España: Universidad Nacional de Educación a Distancia. Recuperado de http://www.quadernsdigitals.net/datos web/biblioteca/l 1400/enLinea/10.pdf

Hernández, D. y Pérez, J. (2014). La autorregulación y la autoevaluación como parte de la estrategia de aprendizaje en los cursos obligatorios que ofrece el Centro de Capacitación de Educación a Distancia. Recuperado de: http://investiga.uned.ac.cr/revistas/index.php/revistacalidad/article/view/684/ $\underline{571}$

Murillo, J. (2013). El arte de aprender cómo se aprende. San José: EUNED.

UNESCO (1988). Un modelo educativo centrado en el aprendizaje. Recuperado de http://sitios.itesm.mx/va/dide/modelo/libro/capitulos espanol/pdf/cap 2.pdf

Universidad Estatal a Distancia (2005). Creación y Organización de la UNED. San José: EUNED.

Universidad Estatal a Distancia (2004). Modelo Pedagógico de la Universidad Estatal a Distancia. Aprobado por el Consejo Universitario, sesión N. ${ }^{\circ}$ 1714, artículo IV, inciso 3) del 9 de julio de 2004. San José: EUNED.

Universidad Estatal a Distancia. (2013a). Plan de Desarrollo Académico 2012 2017. Recuperado de http://www.uned.ac.cr/academica/plan academico/insumos/PlanDesAcad UNED final.pdf

Valle, A., González, R., Núñez, J., Suárez, M., Piñeiro, I. y Rodríguez, S. (2000). Enfoques de aprendizaje en estudiantes universitarios. Recuperado de http://www.unioviedo.es/reunido/index.php/PST/article/view/7605/7469 